\title{
High-Performance Repetitive Control of PWM DC-AC Converters With Real-Time Phase-Lead FIR Filter
}

\author{
Yongqiang Ye, Keliang Zhou, Bin Zhang, Danwei Wang, and Jingcheng Wang
}

\begin{abstract}
The significance of phase-lead compensation is revealed for repetitive control systems in terms of tracking accuracy and transient. A real-time noncausal phase-lead FIR filter is proposed to improve the performance of add-on repetitive controlled constant-voltage constant-frequency PWM dc-ac converters. The experiment results show that nearly perfect tracking, low total harmonics distortion, and satisfactory transient are achieved in the proposed repetitive-controlled PWM converter under both linear load and rectifier load.
\end{abstract}

Index Terms-Constant-voltage constant frequency (CVCF) pulsewidth-modulated (PWM) dc-ac converters, minimized total harmonics distortion (THD), noncausal finite-impulse-response (FIR) filter, repetitive control.

\section{INTRODUCTION}

$\mathbf{C}$ ONSTANT-VOLTAGE constant-frequency (CVCF) pulsewidth-modulated (PWM) converters are widely employed in ac power conditioning systems. High-performance CVCF PWM converters should regulate the output ac voltage to the sinusoidal reference with low total harmonic distortion (THD) and satisfactory dynamic response. Nonlinear loads, such as rectifier loads, causing periodic distortion, are major sources of THD.

Several high-precision feedback control schemes, such as deadbeat or one-sampling-ahead-preview (OSAP) controller [1], [2], sliding mode controller (SMC) [3], and hysteresis controller (HC) [4], are proposed to minimize THD. Unfortunately, feedback control alone cannot eliminate the periodic distortion caused by nonlinear loads and parameter uncertainties.

Repetitive control (RC) [5], [6] is a specialized feedforward control strategy for improving control accuracy by adjusting the control input periodically based on the errors of previous pe-

Manuscript received August 10, 2005. This work was supported in part by the Zhejiang Provincial Natural Science Foundation of China under Grant Y105004 and by the Zhejiang Provincial Scientific Research Foundation for the Returned Overseas Chinese Scholars (No. 15-32). The work of J. Wang was supported by the National Natural Science Foundation of China under Grant 60374011. This paper was recommended by Associate Editor X. Xia.

Y. Ye is with the School of Information, Zhejiang University of Finance and Economics, Hangzhou 310012, China (e-mail: yongqiang_leaf@ hotmail.com).

K. Zhou is with the Delft University of Technology, 2628 CD, Delft, The Netherlands (e-mail: eklzhou@ieee.org).

B. Zhang was with the Nanyang Technological University, Singapore 639798. He is now with the Georgia Institute of Technology, Atlanta, GA 30332 USA.

D. Wang is with Nanyang Technological University, Singapore 639798 (e-mail: edwwang@ntu.edu.sg).

J. Wang is with the Department of Automation, Shanghai Jiaotong University,

Shanghai 200240, China (e-mail: jcwang@sjtu.edu.cn).

Digital Object Identifier 10.1109/TCSII.2006.875383 $\operatorname{riod}(\mathrm{s})$. RC can improve the steady-state tracking accuracy of CVCF PWM converters [7]-[9].

Phase-lead compensation, especially zero-phase compensation, plays a critical role in improving the tracking accuracy and convergence rate. A noncausal filter is used to offer phase lead and to achieve an overall phase of zero. For simplicity of online calculation, the noncausal filter is truncated to form a finite-impulse response (FIR) filter. The real-time zero-phase repetitive controller is successful applied to a CVCF PWM dc-ac converter. Almost perfect tracking accuracy, low THD, and satisfactory tracking transient are achieved under parameter uncertainties and nonlinear load.

\section{Phase-Lead RC}

\section{A. Add-On RC}

Feedback control and RC are complementary. Feedback control emphasizes on dynamic response, while $\mathrm{RC}$ specializes in improving steady-state tracking accuracy. Combination of RC and feedback control yields a high-performance control scheme.

$\mathrm{RC}$ is added outside the closed loop and simply adjusts the command given to the existing feedback control system, as shown in Fig. 1. In Fig. 1, $Y_{d}(z)$ is the periodic reference signal of period $N, Y(z)$ is the output, $D(z)$ is the periodic disturbance of period $N, E(z)$ is the tracking error, $G_{s}(z)$ is the plant, and $G_{c}(z)$ is the feedback controller, $G_{r}(z)$ is the repetitive controller. Denote the closed-loop transfer function $H(z)=G_{c}(z) G_{s}(z) /\left[1+G_{c}(z) G_{s}(z)\right]$.

Fig. 2 shows the repetitive controller $G_{r}(z)$. The RC law in $G_{r}(z)$ can be written as

$$
U_{r}(z)=Q(z)\left[z^{-N} U_{r}(z)+k_{r} z^{-N} G_{f}(z) E(z)\right]
$$

where $N=f_{c} / f$ is the period where $f_{c}$ is the sampling frequency and $f$ is the reference signal frequency, $k_{r}$ is the repetitive control gain, and $G_{f}(z)$ is a filter. $Q(z)$ is a low-pass zero-phase filter. $Q(z)=\left(d_{1} z+d_{0}+d_{1} z^{-1}\right)$ is generally sufficient, with $d_{0}+2 d_{1}=1$. $Q(z)$ brings a tradeoff between tracking accuracy and the system robustness.

It is well known that the stability condition of the $\mathrm{RC}$ is [6]

$$
\begin{aligned}
\left|Q(z)\left(1-k_{r} G_{f}(z) H(z)\right)\right|<1 \quad \forall z= & e^{j \omega}, \\
& 0<\omega<\pi .
\end{aligned}
$$

\section{B. Phase-Lead FIR Filter in RC}

If $Q(z)=1$, (2) becomes

$$
\left|1-k_{r} G_{f}\left(e^{j \omega}\right) H\left(e^{j \omega}\right)\right|<1 \quad \forall 0<\omega<\pi .
$$




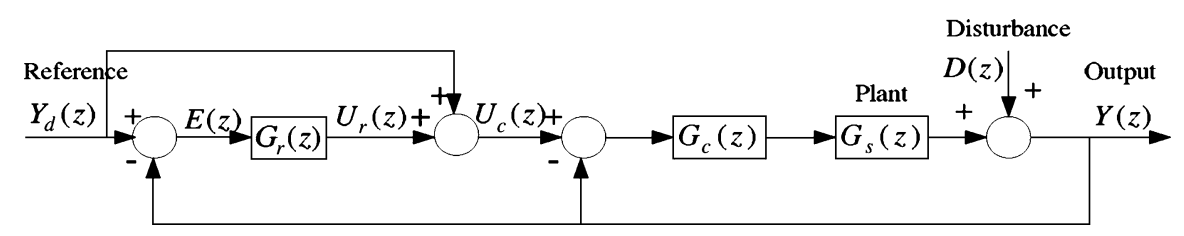

Fig. 1. RC system.

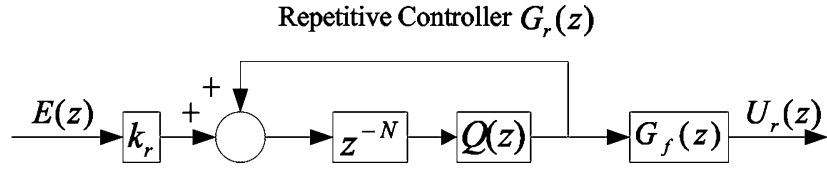

Fig. 2. Repetitive controller.

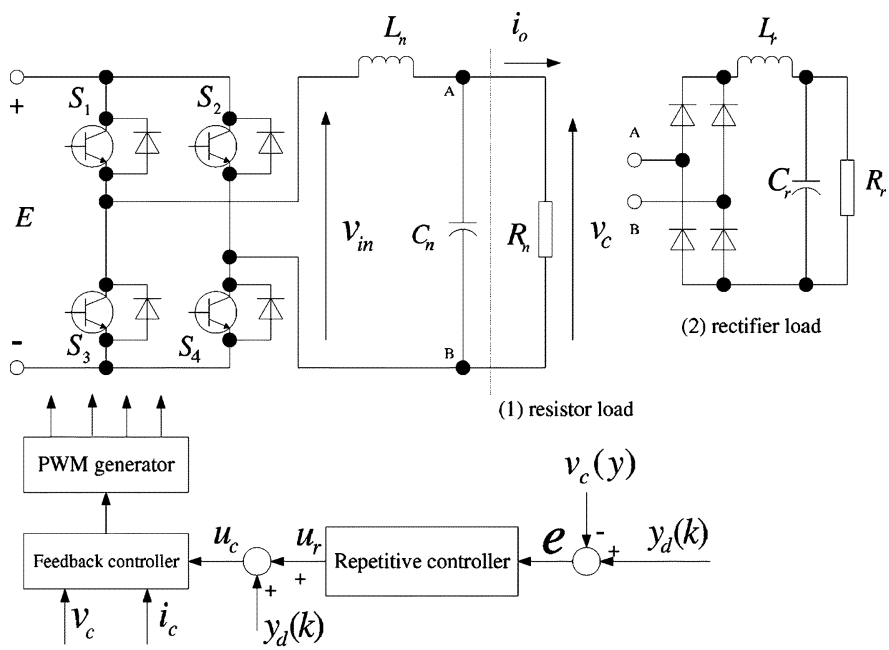

Fig. 3. CVCF PWM dc-ac converter.

If (3) is satisfied, zero steady-state tracking error can be obtained. For a dc-ac converter, $H(z)$ varies within a restricted region under parameter uncertainties and nonlinear load. The performance of the repetitive controller depends heavily on $G_{f}(z)$.

Suppose $H(z)$ has frequency characteristics $H(j \omega)=$ $N_{h}(\omega) \exp \left(j \theta_{h}(\omega)\right)$, where $N_{h}(\omega)$ and $\theta_{h}(\omega)$ are its magnitude characteristics and phase characteristics, respectively, and $G_{f}(z)$ has frequency characteristics $G_{f}(j \omega)=$ $N_{f}(\omega) \exp \left(j \theta_{f}(\omega)\right)$ where $N_{f}(\omega)$ and $\theta_{f}(\omega)$ are the magnitude characteristics and phase characteristics, respectively. Using these characteristics and noting the fact that $k_{r}$ is generally positive, (3) leads to

$$
k_{r} N_{h}(\omega) N_{f}(\omega)<2 \cos \left(\theta_{h}(\omega)+\theta_{f}(\omega)\right)
$$

Equation (4) indicates that, ideally, $\theta_{f}(\omega)=-\theta_{h}(\omega)$ so that the stability condition becomes $k_{r}<2 /\left(N_{h}(\omega) N_{f}(\omega)\right)$ which is always realizable within the whole frequency band at a satisfactory transient response. In such cases, $G_{f}(z)$ offers phase-lead compensation that is exactly the inverse of the phase of $H(z)$ and achieves the so-called zero-phase effect. In this paper, noncausal filter is employed in RC to get phase lead to approximately eliminate the phase lag of $H(z)$.
Suppose we have a causal filter $F_{c}(z)$ with magnitude characteristic $N_{c}(\omega)$ and phase lag $\theta_{c}(\omega)$. Its noncausal counterpart can be obtained by replacing $z$ with $z^{-1}$ in $F_{c}(z)$, denoted as $F_{c}\left(z^{-1}\right)$. Then, the phase lead of the noncausal filter $F_{c}\left(z^{-1}\right)$ is $-\theta_{c}(\omega)$ while its magnitude characteristics stay unchanged [10]. $F_{c}(z) F_{c}\left(z^{-1}\right)$ achieves the zero-phase effect

$$
F_{c}(z) F_{c}\left(z^{-1}\right)=N_{c}^{2}(\omega) .
$$

Note that $N_{c}^{2}(\omega)$ is real. Therefore, it is desirable that $G_{f}(z)$ is selected as the noncausal counterpart of $H(z)$, i.e., $G_{f}(z)=$ $H\left(z^{-1}\right)$.

$H(z)$ can be represented in Markov parameters as

$$
H(z)=h_{0}+h_{1} z^{-1}+h_{2} z^{-2}+\ldots+h_{i} z^{-i}+\ldots
$$

where $h_{i}, i=0,1, \ldots$ is the Markov parameters (impulse response history). $G_{f}(z)=H\left(z^{-1}\right)$ is

$$
G_{f}(z)=h_{0}+h_{1} z+h_{2} z^{2}+\ldots+h_{i} z^{i}+\ldots
$$

Then, the RC is stable if

$$
0<k_{r}<\min _{\omega} \frac{2}{N_{h}^{2}(\omega)} .
$$

If the series of Markov parameters $h_{i}$ is too long, the computational burden of filtering will be too heavy and possibly the computation cannot be implemented online in experiments. Considering the fact $h_{i}$ decays to zero quickly when $i$ increases, the first $n$ parameters are kept and $G_{f}(z)$ becomes an $n$ th-order FIR noncausal filter

$$
G_{f}(z)=h_{0}+h_{1} z+h_{2} z^{2}+\ldots+h_{n} z^{n}
$$

where $n$ is decided by the specification of tracking accuracy and the hardware capability. The noncausal terms $z^{i}$ can be absorbed by the $N$-steps delay in RC, and the repetitive control law is

$$
u_{r}(k)=u_{r}(k-N)+k_{r} \sum_{i=0}^{n} h_{i} e(k-N+i) \text {. }
$$

Of course, after truncation, $G_{f}(z) H(z)$ cannot exactly achieve the zero-phase effect, whereas the phase restriction for convergence is

$$
-90^{\circ}<\theta_{h}(\omega)-\theta_{c}(\omega)<90^{\circ}
$$

noting that the left-hand side of (4) is positive. Obviously, if $\theta_{h}(\omega)-\theta_{c}(\omega)$ is sufficiently small, we can always find an appropriate gain $k_{r}$ to make the RC system to have a good convergence rate and be stable. Moreover, $Q(z)$ can stabilize the RC at frequencies where (4) is violated [6]. 
TABLE I

PARAMETERS OF EXPERIMENT

\begin{tabular}{|c|c|c|c|c|}
\hline \hline Nominal & Actual & Rectifier Load & Feedback Control & Others \\
\hline$E_{n}=80 \mathrm{~V}$ & $E=70 \mathrm{~V}$ & $C_{r}=470 \mu \mathrm{F}$ & $k_{1}=0.0018$ & $y_{d}(t)=50 \sin 100 \pi t \mathrm{~V}$ \\
\hline$C_{n}=45 \mu \mathrm{F}$ & $C=50 \mu \mathrm{F}$ & $L_{r}=1 \mathrm{mH}$ & $k_{2}=1.67 \times 10^{-6}$ & $f=50 \mathrm{~Hz}$ \\
\hline$L_{n}=20 \mathrm{mH}$ & $L=30 \mathrm{mH}$ & $R_{r}=22 \Omega$ & $r=0.0018$ & $f_{c}=10 \mathrm{kHz}$ \\
\hline$R_{n}=15 \Omega$ & $R=22 \Omega$ & & & \\
\hline
\end{tabular}

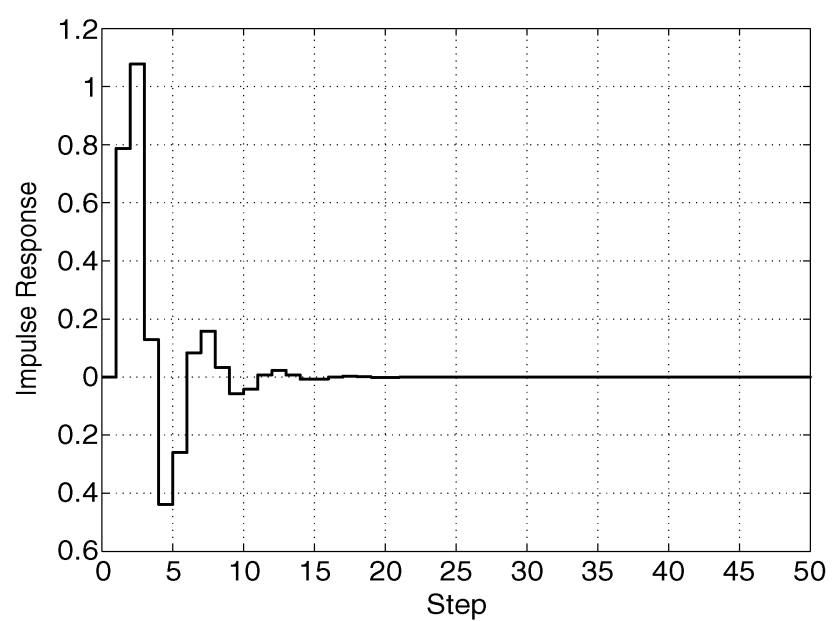

Fig. 4. Impulse response history of $H(z)$.

\section{RC-CONTROLLED DC-AC CONVERTERS}

The dynamics of a CVCF PWM dc-ac converter (as shown in Fig. 3) can be described as follows [1]:

$$
\left[\begin{array}{l}
\dot{v}_{c} \\
\ddot{v}_{c}
\end{array}\right]=\left[\begin{array}{cc}
0 & 1 \\
-\frac{1}{L_{n} C_{n}} & -\frac{1}{C_{n} R_{n}}
\end{array}\right]\left[\begin{array}{l}
v_{c} \\
\dot{v}_{c}
\end{array}\right]+\left[\begin{array}{c}
0 \\
\frac{1}{L_{n} C_{n}}
\end{array}\right] v_{\text {in }}
$$

where $v_{c}$ is the output voltage, $i_{O}$ is the output current, $E$ is the dc bus voltage, and $L_{n}, C_{n}$, and $R_{n}$ are the nominal values of the inductor, capacitor, and load, respectively. The nominal value of $E$ is denoted as $E_{n}$. A sampled-data form for (12) can be expressed as follows [7]:

$$
\left[\begin{array}{l}
v_{c}(k+1) \\
\dot{v}_{c}(k+1)
\end{array}\right]=\left[\begin{array}{ll}
\varphi_{11} & \varphi_{12} \\
\varphi_{21} & \varphi_{22}
\end{array}\right]\left[\begin{array}{l}
v_{c}(k) \\
\dot{v}_{c}(k)
\end{array}\right]+\left[\begin{array}{l}
g_{1} \\
g_{2}
\end{array}\right] v_{\text {in }}(k)
$$

where coefficients $\varphi_{11}=1-\left(T^{2} / 2 L_{n} C_{n}\right), \varphi_{21}=$ $-\left(T / L_{n} C_{n}\right)+\left(T^{2} / 2 L_{n} C_{n}^{2} R_{n}\right), \varphi_{12}=T-\left(T^{2} / 2 C_{n} R_{n}\right)$, $\varphi_{22}=1-\left(T / C_{n} R_{n}\right)-\left(T^{2} / 2 L_{n} C_{n}\right)+\left(T^{2} / 2 C_{n}^{2} R_{n}^{2}\right)$, $g_{1}=\left(T^{2} / 2 L_{n} C_{n}\right)$, and $g_{2}=\left(T / L_{n} C_{n}\right)\left(1-\left(T / 2 C_{n} R_{n}\right)\right)$. The objective is to force the tracking error between $y(k)=v_{c}(k)$ and its sinusoidal reference $y_{d}(k)$ of period $N$ to approach zero asymptotically.

When a state-feedback control scheme

$$
v_{\text {in }}(k)=\left[-k_{1}-k_{2}\right]\left[\begin{array}{l}
v_{c}(k) \\
\dot{v}_{c}(k)
\end{array}\right]+r u_{c}(k)
$$

where $u_{c}(k)$ is a new input variable, is introduced, (13) becomes

$$
\begin{array}{r}
{\left[\begin{array}{c}
v_{c}(k+1) \\
\dot{v}_{c}(k+1)
\end{array}\right]=\left[\begin{array}{lr}
\varphi_{11}-k_{1} g_{1} & \varphi_{12}-k_{2} g_{1} \\
\varphi_{21}-k_{1} g_{2} & \varphi_{22}-k_{2} g_{2}
\end{array}\right]\left[\begin{array}{l}
v_{c}(k) \\
\dot{v}_{c}(k)
\end{array}\right]} \\
+\left[\begin{array}{l}
r g_{1} \\
r g_{2}
\end{array}\right] u_{c}(k) .
\end{array}
$$

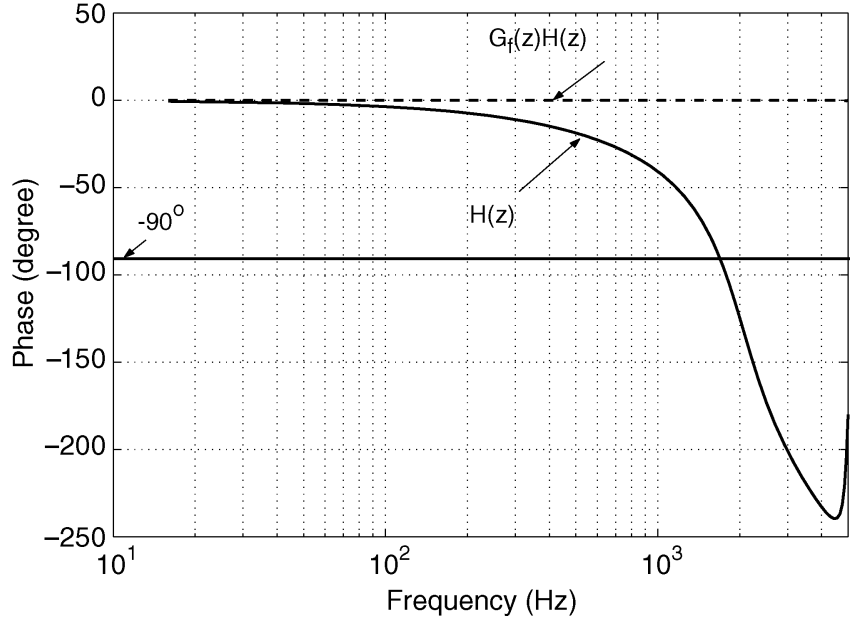

Fig. 5. Phase before and after compensation.

Note that $u_{c}(k)$ is the command input, with the $z$-transform $U_{c}(z)$ indicated in Fig. 1. The transfer function from $u_{c}(k)$ to $y(k)$ can be derived as

$$
H(z)=\frac{m_{1} z^{-1}+m_{2} z^{-2}}{1+p_{1} z^{-1}+p_{2} z^{-2}}
$$

where $m_{1}=r g_{1}, m_{2}=r g_{2}\left(\varphi_{12}-k_{2} g_{1}\right)-r g_{1}\left(\varphi_{22}-k_{2} g_{2}\right)$, $p_{1}=-\left(\varphi_{11}-k_{1} g_{1}-\left(\varphi_{22}-k_{2} g_{2}\right)\right.$, and $p_{2}=\left(\varphi_{11}-\right.$ $\left.k_{1} g_{1}\right)\left(\varphi_{22}-k_{2} g_{2}\right)-\left(\varphi_{12}-k_{2} g_{1}\right)\left(\varphi_{21}-k_{1} g_{2}\right)$.

Note that the response of the feedback-controlled converter will deviate from the designated one because of disturbances $\Delta R=R-R_{n}$ and uncertainties $\Delta L=L-L_{n}, \Delta C=$ $C-C_{n}$, and $\Delta E=E-E_{n}$. To improve the performance of the state-feedback control, RC law (10) is employed.

\section{EXPERIMENTS}

\section{A. Experiment Parameters}

The parameters of the converter system are listed in Table I. The nominal transfer function $H(z)$ with $R=22 \Omega$ and sampling frequency $10 \mathrm{kHz}$ can be derived as

$$
H(z)=\frac{0.525 z+0.479}{z^{2}-0.4564 z+0.4613} .
$$

$\mathrm{RC}$ gain $k_{r}$ is chosen as 0.8 . The Markov parameters are calculated based on (17). Fig. 4 shows the impulse response history. The first 21 parameters are kept (note that $h_{0}=0$ ). Fig. 5 plots the phase of (17) and the overall phase after the compensation by the noncausal FIR filter $h_{1} z+\ldots+h_{20} z^{20}$. The plot shows that 21 parameters are well enough to achieve nearly perfect zero-phase effect. 


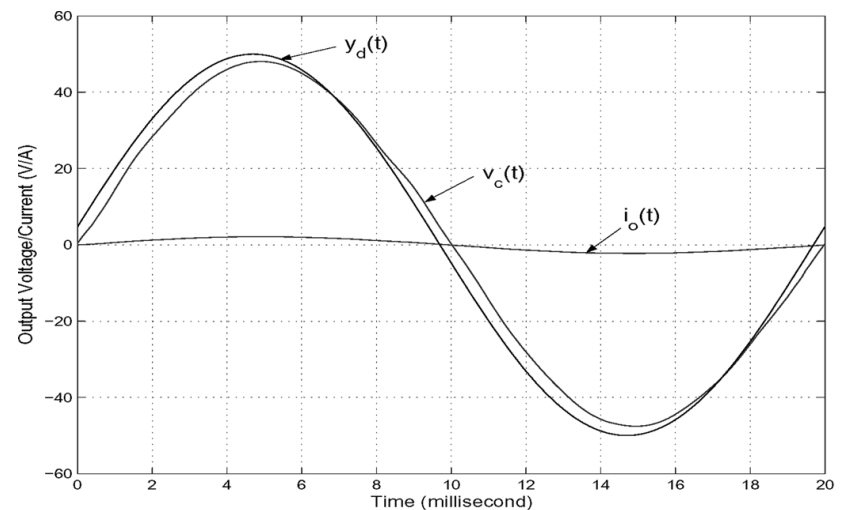

(a)

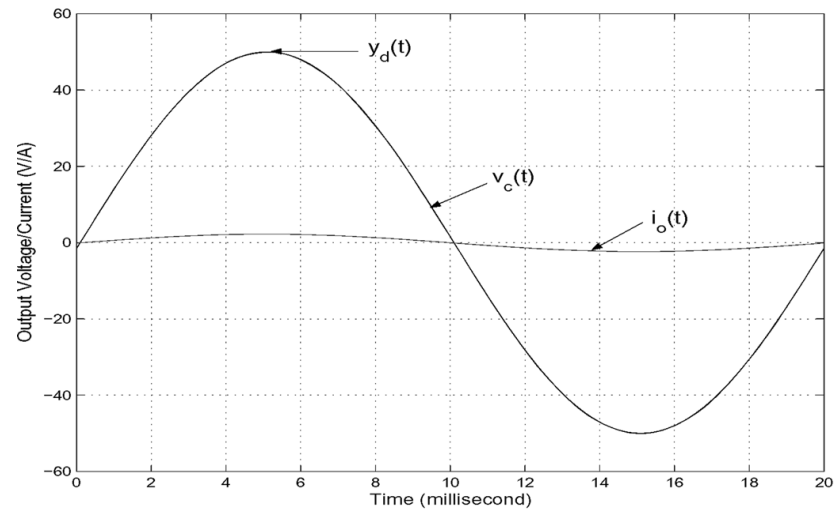

(c)

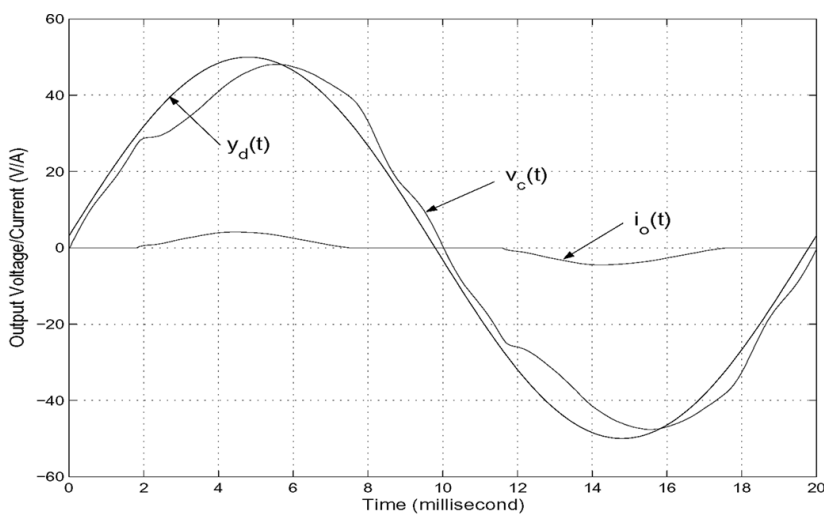

(b)

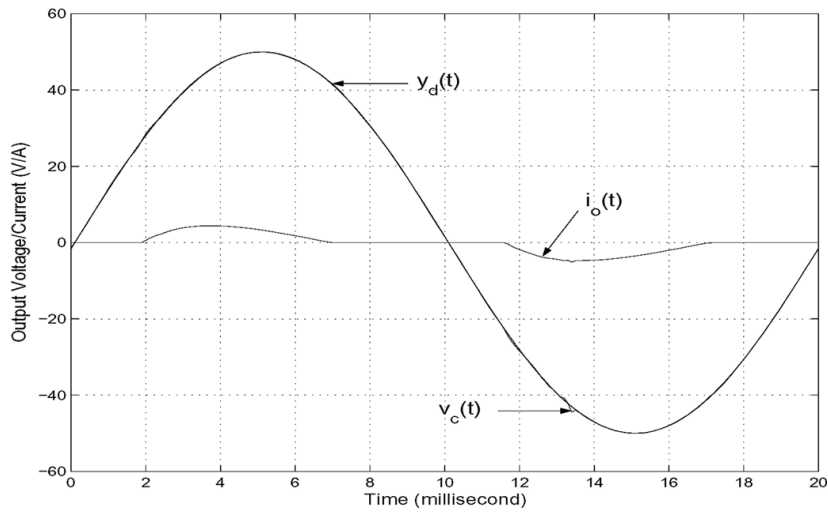

(d)

Fig. 6. Steady-state responses. (a) Feedback control under resistor. (b) Feedback control under rectifier. (c) RC under resistor. (d) RC under rectifier.

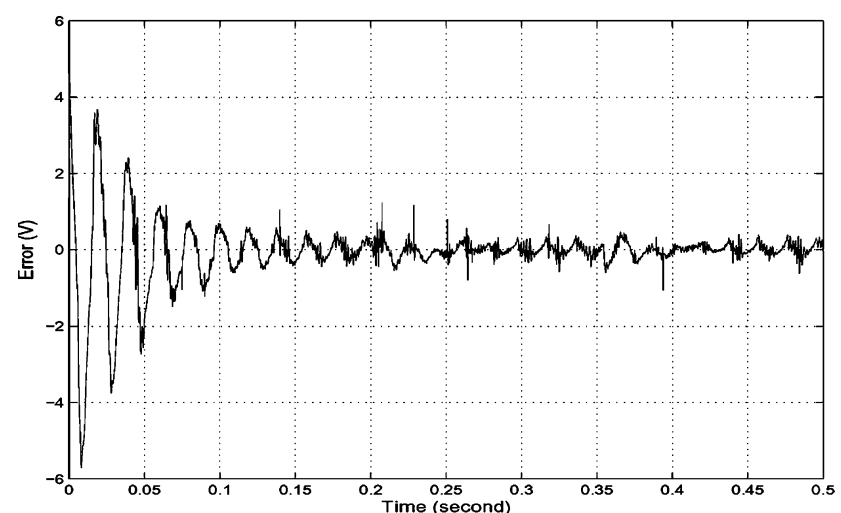

(a)

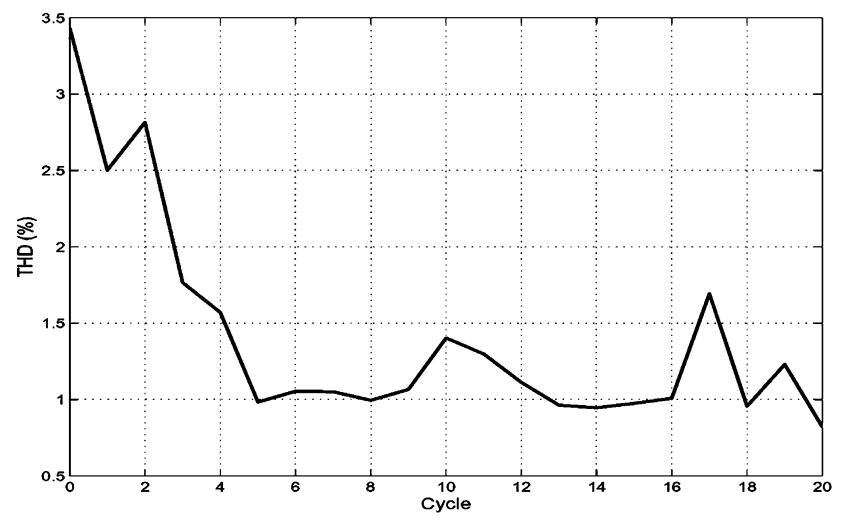

(c)

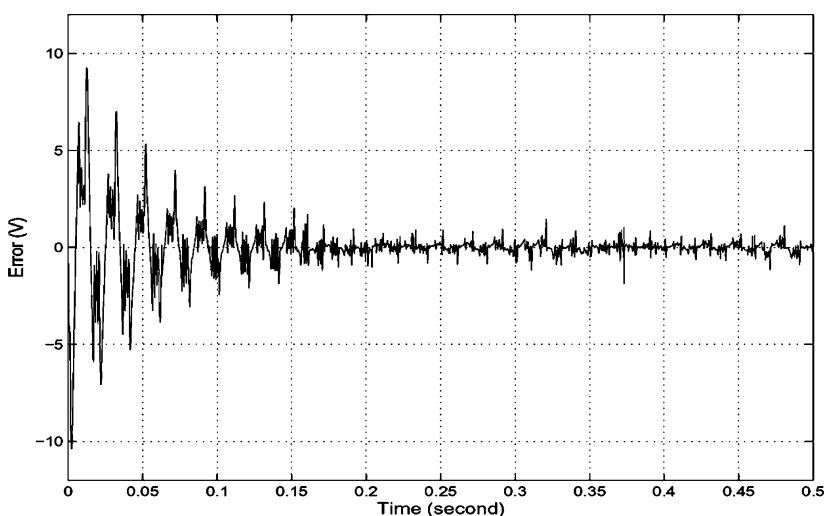

(b)

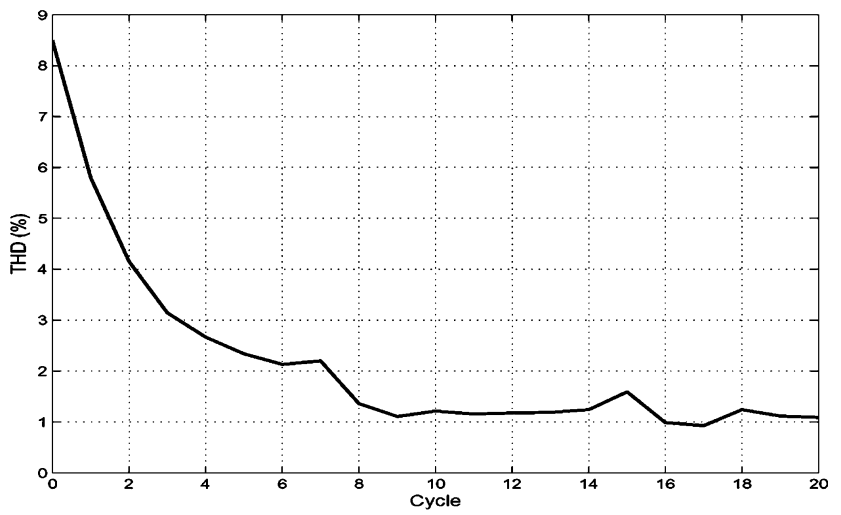

(d)

Fig. 7. Transient performance. (a) Error transient under resistor. (b) Error transient under rectifier. (c) THD history under resistor. (d) THD history under rectifier. 
In the experiments, we found that there is severe unmodeled delay in the system. To tackle against the unmodeled delay, additional linear phase lead [6] is also added to enhance the RC law, and the RC law is modified as

$$
u_{r}(k)=u_{r}(k-N)+k_{r} \sum_{i=1}^{20} h_{i} e(k-N+i+\gamma)
$$

where $\gamma=3$ is the additional lead-steps, i.e., the noncausal filter is shifted three steps forward. $Q(z)=0.25 z^{-1}+0.5+0.25 z$ is used.

\section{B. Experimental Results}

Fig. 6(a) and (b) shows the steady-state responses (SSRs) under resistor load and rectifier load with only feedback control, respectively. The average total harmonic distortions (THDs)of voltage in the two cases are $2.8 \%$ (resistor) and $8.21 \%$ (rectifier), respectively. Fig. 6(c) and (d) shows the RC-controlled SSRs in the two cases. The average THDs are $1.03 \%$ and $1.1 \%$, respectively. Fig. 7(a) and (b) shows the transient responses of tracking error $e(t)=y_{d}(t)-v_{c}(t)$ with $\mathrm{RC}$ in the two cases. The error converges within $0.2 \mathrm{~s}$. Fig. 7(c) and (d) shows the THD versus cycle histories in the two cases. The THDs decrease significantly within 10 cycles.

\section{CONCLUSION}

The significance of phase-lead compensation in RC is highlighted in terms of stability and tracking accuracy. A noncausal FIR filter is employed in RC to offer phase lead to offset phase lag. A noncausal FIR filter constructed from the Markov parameters approximately achieves zero-phase effect. The proposed real-time zero-phase RC scheme is added on a feedback-controlled CVCF PWM dc-ac converter. The RC design is experimentally demonstrated. The periodic tracking errors caused by parameter uncertainties and nonlinear load disturbances are eliminated quite well. With such a real-time phase-lead FIR filter, a high-performance RC-controlled CVCF PWM DC/AC converter is achieved in terms of quick convergence and lowoutput-voltage THD. The scheme is also applicable to threephase converters [11], [12].

\section{REFERENCES}

[1] K. P. Gokhale, A. Kawamura, and R. G. Hoft, "Dead beat microprocessor control of PWM inverter for sinusoidal output waveform synthesis," in Proc. IEEE Power Electron. Specialist Conf., Toulouse, France, Jun. 1985, pp. 28-36.

[2] A. Kawamura and K. Ishihara, "Real time digital feedback control of three phase PWM inverter with quick transient response suitable for uninterruptible power supply," in Proc. Ind. Appl. Soc. Annu. Meeting, Pittsburgh, PA, Oct. 1988, pp. 728-734.

[3] M. Carpita and M. Marchesoni, "Experimental study of a power conditioning system using sliding mode control," IEEE Trans. Power Electron., vol. 11, no. 5, pp. 731-733, May 1996.

[4] A. Kawamura and R. G. Hoft, "Instantaneous feedback controlled PWM inverter with adaptive hysteresis," IEEE Trans. Ind. Appl., vol. 20, no. 4, pp. 769-775, Oct. 1984.

[5] M. Tomizuka, T. C. Tsao, and K. K. Chew, "Analysis and synthesis of repetitive controllers," ASME J. Dyn. Syst., Meas., Control, vol. 111, no. 3, pp. 353-358, Sep. 1989.

[6] R. W. Longman, "Iterative learning control and repetitive control for engineering practice," Int. J. Control, vol. 73, no. 10, pp. 930-954, 2000.

[7] K. Zhou and D. Wang, "Periodic errors elimination in CVCF PWM DC/AC converter systems: Repetitive control approach," Proc. IEE Control Theory Appl., vol. 147, no. 6, pp. 694-700, Nov. 2000.

[8] T. Haneyoshi, A. Kawamura, and R. G. Hoft, "Waveform compensation of PWM inverter with cyclic fluctuating loads," IEEE Trans. Ind. Appl., vol. 24, no. 4, pp. 582-589, Oct. 1988.

[9] G. Weiss, Q. Zhong, T. Green, and J. Liang, “ $\mathrm{H}^{\infty}$ repetitive control of DC-AC converters in microgrids," IEEE Trans. Power Electron., vol. 19, no. 1, pp. 219-230, Jan. 2004

[10] A. V. Oppenheim and R. W. Schafer, Discrete-Time Signal Processing. Englewood Cliffs, NJ: Prentice-Hall, 1989.

[11] K. Zhou and D. Wang, "Digital repetitive learning controller for threephase CVCF PWM inverter," IEEE Trans. Ind. Electron., vol. 48, no. 4, pp. 820-830, Aug. 2001.

[12] — , "Digital repetitive controlled three-phase PWM rectifier," IEEE Trans. Power Electron., vol. 18, no. 1, pp. 309-316, Jan. 2003. 